\title{
ESTUDIO DE BIODEGRADABILIDAD AERÓBICA DE HDPE CON ADITIVOS DEGRADABLES MEDIANTE COMPOSTAJE
}

\author{
STUDY OF AEROBIC BIODEGRADABILITY OF HDPE \\ WITH DEGRADABLE ADDITIVES BY COMPOSING
}

\section{Ma. Judith Terán S., ${ }^{1}$ David Romero E. ${ }^{2}$ \& Lorena Meneses O. ${ }^{1}$}

Recibido: 26 septiembre 2017 / Aceptado: 12 diciembre 2017

Palabras claves: biodegradabilidad, compostaje, materia orgánica, microorganismos, polietileno de alta densidad, suelo. Keywords: biodegradability, composting, high density polyethylene, microorganisms, organic matter, soil.

\section{RESUMEN}

En el presente estudio se estableció el porcentaje de biodegradabilidad de muestras de polietileno de alta densidad (HDPE) con aditivos degradables, mediante condiciones controladas de compostaje aerobio en un período de 90 días, para lo cual se utilizó el $\mathrm{CO}_{2}$ proveniente de procesos biológicos de microorganismos del suelo, como indicador de biodegradabilidad. Para captar el $\mathrm{CO}_{2}$ se emplearon soluciones de $\mathrm{KOH} \mathrm{0,1} \mathrm{M} \mathrm{y} \mathrm{0,5} \mathrm{M.} \mathrm{Además} \mathrm{se} \mathrm{establecieron}$ los parámetros de análisis estipulados en la norma NTE INEN 2040:2012 para seleccionar el inóculo de compostaje. Como control positivo se utilizó celulosa, 
que alcanzó el 91 \% de biodegradabilidad con respecto al valor teórico de $\mathrm{CO}_{2}$. Los resultados obtenidos al concluir el período de 90 días, indicaron que la muestra de HDPE con aditivo A1 (HDPE-A1) alcanzó el 75 \% de biodegradabilidad por generación de $\mathrm{CO}_{2}$, seguido del HDPE-A2 con el $43 \%$ y el HDPE-A3 con el $22 \%$, con respecto a la celulosa. Los valores demuestran que el aditivo A1 es biodegradable en condiciones de compostaje, lo que se estableció mediante el criterio de la norma NTE INEN 2643:2012 "Especificaciones para plásticos compostables". Los resultados demuestran que los aditivos biodegradables son una posible solución a la gran problemática ambiental que han generado los plásticos.

\section{ABSTRACT}

The study determinate the percentage of biodegradability of samples of high density polyethylene (HDPE) with degrading additives, in controlled conditions in a period of 90 days for which the generation of $\mathrm{CO}_{2}$ from biological processes of soil microorganisms was used as an indicator of biodegradability. For the absorption of $\mathrm{CO}_{2}$ were used solutions of $0.1 \mathrm{M}$ and $0.5 \mathrm{M} \mathrm{KOH}$. In addition, the analysis parameters stipulated in the standard NTE INEN 2040: 2012 were established to select the composting inoculum. As a positive control was used cellulose which reached $91 \%$ of biodegradability with respect to the theoretical value of $\mathrm{CO}_{2}$ that this material can generate. The results obtained at the end of the 90 days, indicated that the HDPE sample with A1 additive reached $75 \%$ biodegradability by $\mathrm{CO}_{2}$ generation, followed by HDPE-A2 with $43 \%$ and HDPE-A3 with $22 \%$ with respect to cellulose. The values show that the additive A1 is biodegradable under composting conditions, this was established by the criterion of the standard NTE INEN 2643: 2012 "Specifications for compostable plastics". The results show that the biodegradable additives are a possible solution to the great environmental problem that have generated the plastics. 


\section{INTRODUCCIÓN}

La industria de los polímeros plásticos en el Ecuador es considerada una de las más dinámicas, ya que de acuerdo con el Gobierno Nacional, es uno de los nueve sectores más importantes para el cambio de la matriz productiva (El Telégrafo, 2015).

En vista que la producción de polímeros plásticos se encuentra en aumento, es necesario tomar medidas respecto al reciclaje y manejo de los productos de esta industria, para reducir su efecto en el ambiente (Ecuador al día, 2014). Pero su disposición final enfocada en el reciclaje como parte de la solución, no es una salida completamente eficaz y su incineración genera compuestos tóxicos, que son altamente contaminantes.

Muchos plásticos que se depositan en rellenos municipales y océanos, provocan la asfixia de animales al consumirlos por accidente. Por esta razón, se han desarrollado compuestos denominados aditivos, que se acoplan a los polímeros y facilitan la degradación de los plásticos en distintas condiciones (Capuz \& Gómez, 2002).
Organismos internacionales como la American Society of Testing Materials (ASTM) e International Organization for Standardization (ISO), elaboraron métodos normados para estudiar la degradación y biodegradación de materiales plásticos, sometiéndolos a distintas condiciones. Normas como la ASTM D5338, D5209 y D5271, miden la generación de $\mathrm{CO}_{2}$ proveniente de procesos metabólicos de microorganismos, como indicador de biodegradabilidad (ASTM D6954, 2004). La aplicación de estos métodos permite establecer si los materiales de ensayo cumplen con las especificaciones para denominarse como plástico degradable o biodegradable.

Se sabe que los microorganismos utilizan compuestos que provienen de la fragmentación de los polímeros y pueden atravesar la pared celular para mineralizarlos mediante enzimas internas. Los microorganismos presentes en el suelo y en el compost son propensos a degradar compuestos poliméricos, debido a la presencia de enzimas y metabolitos (Ammala, 2011). 
Generalmente se ha empleado el mecanismo de hidroperoxidación de los materiales plásticos como posible vía de descomposición, ya que el radical hidroperóxil es organosoluble y capaz de reaccionar con enlaces carbono-hidrógeno de los polímeros (Chiellini \& Solaro, 2003; San Andrés, 2010).

El compostaje consiste en la descomposición biológica de residuos orgánicos, donde están involucrados una amplia variedad de microorganismos. Consta de cuatro fases (mesofílica, termofílica, enfriamiento y maduración); la fase termofílica aporta el mayor grado de descomposición. Esta fase se caracteriza por la presencia de bacterias termofílicas y actinomicetos, la temperatura se encuentra entre 50 y $60{ }^{\circ} \mathrm{C}$ y permite eliminar bacterias y contaminantes fecales como E. coli y Salmonella spp, al igual que quistes, huevos de helmintos y esporas de hongos fitopatógenos, así se asegura un producto higienizado (Kalil, 2007).

El inóculo que se utiliza en el compostaje, consiste en un suelo que funciona como un agente para la biodegradación, los factores de control en este suelo son: la relación carbono nitrógeno $(\mathrm{C} / \mathrm{N})$, el $\mathrm{pH}$, la temperatura, la humedad, el tamaño de partícula del suelo y oxígeno (FAO, 2003; Román, Martínez \& Pantoja, 2013).

El material considerado en este estudio es polietileno de alta densidad (HDPE), al que se le han añadido aditivos degradables. En vista que las muestras analizadas son comerciales y están protegidas por el derecho industrial, la única información disponible con que se cuenta, es que el aditivo A1 es biodegradable, y los aditivos A2 y A3 son oxobiodegradables. Debido a la disposición final de estos plásticos, es necesario determinar cuál de las muestras se descompone con mayor facilidad, bajo condiciones de compostaje aerobio. Por lo tanto, el objetivo principal de la investigación fue estudiar la biodegradabilidad de polietileno de alta densidad con aditivos A1, A2 y A3 en condiciones controladas de compostaje aerobio, para determinar el porcentaje de biodegradabilidad en un período de 90 días. 


\section{MATERIALES Y MÉTODOS}

La metodología empleada se basó en la norma NTE INEN 2640:2012 "Método de ensayo para determinar la biodegradabilidad aeróbica de materiales plásticos bajo condiciones controladas de compostaje" (Norma Técnica Ecuatoriana INEN 2640: 2012).

\section{Toma de muestras}

Las muestras fueron proporcionadas por el Centro de Servicios Ambientales y Químicos de la Pontificia Universidad Católica del Ecuador (CESAQ-PUCE), que estudia el comportamiento de las fundas plásticas en condiciones controladas de compostaje aerobio. Las muestras consistieron en fundas plásticas tipo camiseta, de polietileno de alta densidad con los aditivos A1, A2 y A3, empleadas en el empaque y trasporte de productos de supermercado.

\section{Ensayos preliminares}

Para establecer las condiciones de compostaje para las muestras en estudio, se realizaron los siguientes ensayos:

\section{a. Análisis en materiales plásticos y celulosa}

Se determinó por gravimetría el contenido de humedad de los materiales plásticos y celulosa, mediante tratamiento térmico a $105{ }^{\circ} \mathrm{C}$ en una estufa marca Memert, modelo SM 200. Se evaluó también el contenido de materia orgánica y ceniza en un horno mufla marca Tecnodalvo, modelo TDHM2, a $550{ }^{\circ} \mathrm{C}$.

Se estableció el coeficiente de variación para cada determinación de materia orgánica, y se tomó en cuenta que los valores de cenizas se establecen a partir del contenido de materia orgánica.

\section{Determinación de condiciones de trampas de $\mathrm{CO}_{2}$}

Se determinó el tiempo de saturación burbujeando $\mathrm{CO}_{2}$ en soluciones de $\mathrm{NaOH}$ 0,1 M y KOH 0,1 y 0,5 M. Para determinar la eficiencia de captación de las trampas y las adecuadas para una captación de 24 y 72 h, se determinaron la alcalinidad total, alcalinidad por bicarbonatos y alcalinidad por carbonatos. 
Se estableció la eficiencia de captación de $\mathrm{CO}_{2}$ tras conectar en serie las trampas de $\mathrm{KOH} 0,1 \mathrm{M}$ y 0,5 M.

De esta manera se seleccionaron las soluciones adecuadas para la captación de $\mathrm{CO}_{2}$. El método de análisis empleado fue el de alcalinidad 2320 B del Standard Methods for the Examination of Water \& Wastewater, el cual usa la volumetría como técnica de análisis (APHA, 2005).

\section{b. Análisis de suelos}

El inóculo de compostaje debe cumplir con los parámetros mencionados en la norma NTE INEN 2640:2012.

Dichos parámetros son: $\mathrm{pH}$ cuya determinación se efectuó por potenciometría empleando un potenciómetro $\mathrm{HACH}$, modelo sensION; la humedad, cenizas y materia orgánica, determinadas por gravimetría, empleando una estufa Memert, modelo SM 200 y un horno mufla Tecnodalvo, modelo TDHM2.

Para la determinación de la cantidad de $\mathrm{CO}_{2}$ por gramo de materia orgánica se sometió el suelo (300 y 600 g), a condiciones de compostaje ae- robio por 10 días $\left(58 \pm 2{ }^{\circ} \mathrm{C}, 50 \%\right.$ de humedad y $2 \mathrm{~L} / \mathrm{min}$ de aireación) y se efectuaron determinaciones de alcalinidad en las soluciones captadoras de $\mathrm{CO}_{2}$.

Para la selección del inóculo, se analizaron cuatro muestras de compost: una muestra de compost de champiñón, constituida por la mezcla de un suelo Entisol con champiñones; una muestra de tierra negra de taxonomía Mollisol; y dos muestras más denominadas compost 1 y 2 que consistían en un Entisol e Inceptisol respectivamente. La taxonomía del suelo se definió con el mapa de suelos de Pichincha (Ministerio de Agricultura, Ganadería, Acuacultura y Pesca, 2012).

\section{Determinación de biodegradabilidad de polietileno de alta densidad me- diante compostaje}

El material plástico y la celulosa se cortaron en trozos de $2 \mathrm{~cm} \times 2 \mathrm{~cm}$. Se pesaron $100 \mathrm{~g}$ de cada material y 600 g del inóculo (relación 6:1), se ajustó el contenido de humedad al $50 \%$ y se sometió a una temperatura de $58 \pm 2{ }^{\circ} \mathrm{C}$ con la ayuda de un baño termostático diseñado para la capa- 
cidad de los recipientes, los cuales consistieron en erlenmeyers de $1 \mathrm{~L}$. Cada 24 h se tomaron alícuotas de las soluciones de $\mathrm{KOH}$ (captadoras de $\mathrm{CO}_{2}$ ), para establecer la cantidad de $\mathrm{CO}_{2}$ retenido. Para conformar el blanco de ensayo se emplearon únicamente 600 g del inóculo.

El aire que ingresó en el sistema de compostaje debía encontrarse libre de $\mathrm{CO}_{2}$ en todo momento.

Ensayos de suelos después del compostaje

Se analizaron los suelos después del proceso de biodegradación de los materiales poliméricos analizados, para lo cual se efectuaron los ensayos indicados en la selección del inóculo, y adicionalmente a estos, se estableció el contenido de carbono orgánico total, en el que se empleó un factor tomando en cuenta que la materia orgánica contiene el 83,5\% de carbono orgánico (Carreira, 2011). Se calculó el coeficiente de variación para cada determinación de materia orgánica y contenido de humedad.

También se determinó el contenido de nitrógeno total, para lo cual se usó un espectrofotómetro UV/vis $\mathrm{HACH}$ DR 2010 y un kit de nitrógeno total $\mathrm{HACH}$ para rango alto.

\section{RESULTADOS}

\section{Ensayos preliminares}

\section{- Análisis en materiales de ensayo y condiciones de trampas de $\mathrm{CO}_{2}$}

En la Tabla 1 se observan los valores obtenidos para materia orgánica, cenizas y humedad al final del tratamiento térmico en HDPE aditivado y celulosa.
Los valores de porcentajes de coeficiente de variación para las determinaciones de materia orgánica para cada material polimérico fueron de 0,63\% HDPE-A1, 2,03\% HDPE-A2, $0,68 \%$ HDPE-A3 y $0,97 \%$ celulosa. 
Tabla 1. Porcentaje de cenizas, humedad y materia orgánica en materiales plásticos analizados y celulosa

\begin{tabular}{lccc}
\hline Muestras & $\begin{array}{c}\text { MO } \\
(\%)\end{array}$ & $\begin{array}{c}\text { Cenizas } \\
(\%)\end{array}$ & $\begin{array}{c}\text { H. } \\
(\%)\end{array}$ \\
\hline HDPE-A1 & 98,45 & 1,55 & 1,42 \\
HDPE-A2 & 97,53 & 2,47 & 1,24 \\
HDPE-A3 & 98,17 & 1,83 & 1,32 \\
Celulosa & 98,16 & 1,84 & 2,02 \\
\hline
\end{tabular}

MO: Materia orgánica

$\mathrm{H}$ : Humedad

- Estudio de saturación de trampas de $\mathrm{CO}_{2}$ alcalinidad en el tiempo, para los tres ensayos realizados.

En la Figura 1 se muestran los resulta- En la Figura 2 se encuentran los redos de la determinación de alcalinisultados de alcalinidad por carbonadad por hidróxidos de las soluciones tos formados en cada solución de hidróxido de sodio 0,1 M e hidrócaptadora, donde la tendencia es el xido de potasio 0,1 y $0,5 \mathrm{M}$, utilizaaumento de la alcalinidad con el das como trampas de $\mathrm{CO}_{2}$. Se puede tiempo. ver una tendencia decreciente de la

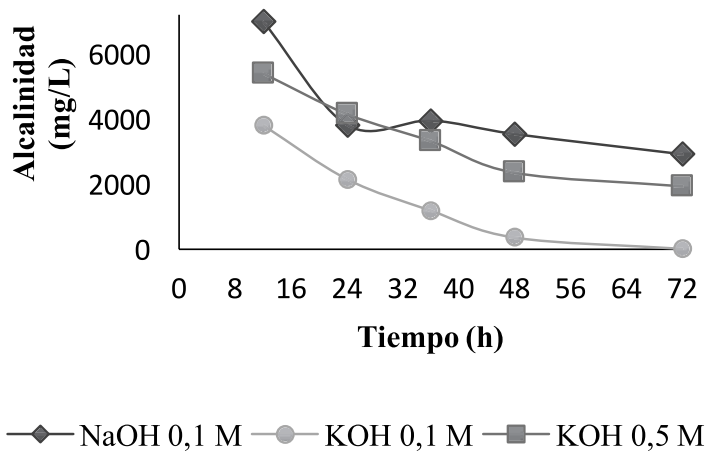

Figura 1. Relación de la alcalinidad por hidróxidos con el tiempo 


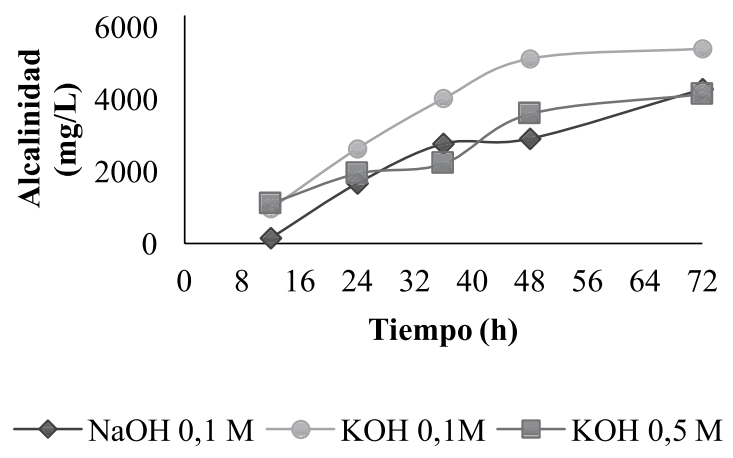

Figura 2. Relación de la alcalinidad por carbonatos con el tiempo

- Prueba de eficiencia de las tramciencia para las tres trampas de $\mathrm{KOH}$ pas de $\mathrm{CO}_{2}$ $0,1 \mathrm{M}$ y $0,5 \mathrm{M}$.

En la Figura 3 se muestran los resultados obtenidos en la prueba de efi-

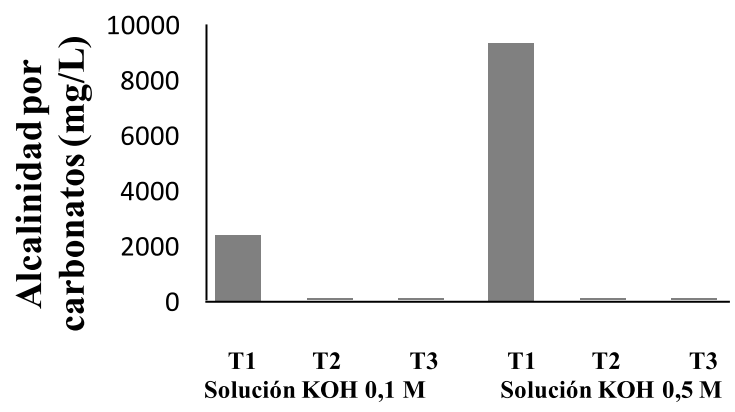

Figura 3. Resultados de alcalinidad por carbonatos para eficiencia de trampas 
- Selección del Inóculo (suelo de compostaje)

En la Tabla 2 se encuentran los resultados de los parámetros obtenidos por gravimetría, en las muestras de suelos utilizados como inóculo de compostaje.

Tabla 2. Valores de pH, materia orgánica, humedad, cenizas y carbono orgánico total en muestras de inóculo

\begin{tabular}{lccccc}
\hline Suelos & pH & $\begin{array}{c}\text { MO } \\
(\%)\end{array}$ & $\begin{array}{c}\text { C } \\
(\%)\end{array}$ & $\begin{array}{c}\text { COT } \\
(\%)\end{array}$ & $\begin{array}{c}\text { H } \\
(\%)\end{array}$ \\
\hline Compost 1 & 5,9 & - & - & - & - \\
Compost 2 & 6,2 & 32,3 & 67,7 & 27,17 & 32,05 \\
Compost de champ. & 9,5 & 59,53 & 40,47 & 49,71 & 47,03 \\
Tierra Negra & 7,6 & 30,99 & 69,00 & 25,89 & 1,57 \\
\hline
\end{tabular}

COT: Carbono orgánico total

Se determinó el coeficiente de variación en los ensayos de materia orgánica. Para las muestras de Compost de Champiñones, Tierra Negra y Compost 2 los valores fueron de 2,40 $\%, 4,11 \%$ y $1,43 \%$ respectivamente. Para el contenido de humedad los valores fueron 1,03\% para Compost de Champiñones, 1,57 \% Tierra Negra y 4,24\% Compost 2.
En la Tabla 3 se encuentran los resultados de la cantidad de $\mathrm{CO}_{2}$ generado por gramo de materia orgánica en 10 días de ensayo para $600 \mathrm{~g}$ de suelo, nitrógeno total y relación carbono nitrógeno $\mathrm{C} / \mathrm{N}$; donde la muestra de tierra negra fue seleccionada como inóculo de compostaje.

Tabla 3. Resultados de cantidad de $\mathrm{CO}_{2} / \mathrm{g} \mathrm{MO}$, nitrógeno total y relación carbono nitrógeno del inóculo seleccionado

\begin{tabular}{lccc}
\hline Suelo & $\mathrm{mgCO}_{2} / \mathbf{g M O}$ & $\begin{array}{c}\mathbf{N} \text { Total } \\
(\%)\end{array}$ & $\mathbf{C} / \mathbf{N}$ \\
\hline Tierra Negra & 58,92 & 0,68 & 38,34 \\
\hline
\end{tabular}


Biodegradabilidad de polietileno de dos acumulados de $\mathrm{CO}_{2}$ producido alta densidad aditivado en 90 días de ensayo por cada una de la muestras.

En la Figura 4 se aprecian los resulta-

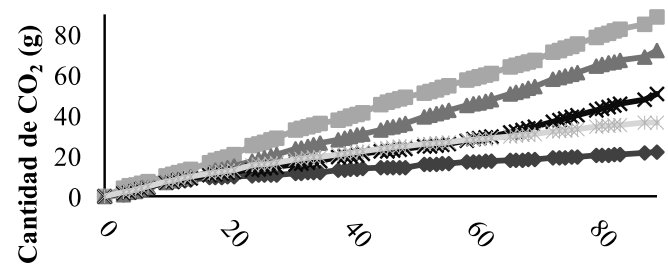

Días de análisis

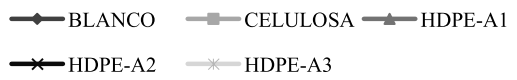

Figura 4. Cantidad de $\mathrm{CO}_{2}$ generado por materiales de ensayo en función del tiempo

La Tabla 4 contiene los resultados del porcentaje de biodegradabilidad (B) obtenidos tomando como $100 \%$ de biodegradabilidad al control positivo (celulosa) en generación de $\mathrm{CO}_{2}$, por comparación con la cantidad de $\mathrm{CO}_{2}$ que teóricamente pueden generar las muestras y la biodegradabilidad por pérdida de masa.

Tabla 4. Biodegradabilidad de materiales plásticos

\begin{tabular}{lccc}
\hline Muestra & $\begin{array}{c}\text { B1 } \\
(\%)\end{array}$ & $\begin{array}{c}\text { B2 } \\
(\%)\end{array}$ & $\begin{array}{c}\text { B3 } \\
(\%)\end{array}$ \\
\hline Celulosa & - & 91 & 91 \\
HDPE-A1 & 75 & 72 & 70 \\
HDPE-A2 & 43 & 41 & 43 \\
HDPE-A3 & 22 & 21 & 21 \\
\hline
\end{tabular}

B1: Biodegradabilidad por generación de $\mathrm{CO}_{2}$

B2: Biodegradabilidad frente al valor teórico

B3: Biodegradabilidad por pérdida de masa 


\section{Ensayos finales en suelos}

En la Tabla 5 se muestran los resultados obtenidos de los parámetros de materia orgánica, cenizas, carbono orgánico total y relación carbono nitrógeno $\mathrm{C} / \mathrm{N}$ para cada suelo empleado como inóculo luego del proceso de compostaje.

Tabla 5. Materia orgánica, cenizas, carbono orgánico total, $\mathrm{pH}, \mathrm{C} / \mathrm{N}$ en suelos después del compostaje

\begin{tabular}{lccccc}
\hline Muestras & $\begin{array}{c}\text { MO } \\
(\%)\end{array}$ & $\begin{array}{c}\text { Cenizas } \\
(\%)\end{array}$ & $\begin{array}{c}\text { COT } \\
(\%)\end{array}$ & C/N & pH \\
\hline Blanco & 18,67 & 81,33 & 15,59 & 35,42 & 7,7 \\
Celulosa & 17,58 & 82,42 & 14,68 & 25,53 & 8,1 \\
HDPE-A3 & 16,05 & 83,95 & 13,40 & 27,08 & 7,7 \\
HDPE-A1 & 16,98 & 83,02 & 14,18 & 25,77 & 8,0 \\
HDPE-A2 & 15,00 & 85,00 & 12,52 & 26,36 & 7,8 \\
\hline
\end{tabular}

El coeficiente de variación obtenido de cada determinación de materia orgánica en los suelos fue de 4,19\% para el blanco de ensayo, $1,46 \%$ celulosa, 2,16\% HDPE-A3, 1,37\% HDPE-A1 y 1,17 \% HDPE-A2.

\section{DISCUSIÓN}

Los resultados obtenidos de los ensayos preliminares, indican que el HDPE con aditivos A1, A2 y A3 contiene material inorgánico en su composición, debido al porcentaje de ceniza, que va del 1,55 al 2,47 \%. El porcentaje de humedad es bajo y el de materia orgánica es alto, que es lo que se espera para un material polimérico.
Las pruebas de tiempo de saturación y eficiencia de trampas de $\mathrm{CO}_{2}$ demostraron que las soluciones de $\mathrm{KOH} \mathrm{0,1} \mathrm{M} \mathrm{y} \mathrm{0,5} \mathrm{M} \mathrm{son} \mathrm{efectivas}$ para captar $\mathrm{CO}_{2}$ ambiental y del sistema de compostaje.

La selección del inóculo es una parte crucial en el ensayo de biodegradabilidad, porque aporta con los microorganismos de biodegradación. El 
suelo escogido como inóculo para este trabajo, fue un Mollisol, colectado en la parroquia de Pifo, que cumplió con las características indicadas por la norma NTE INEN 2640:2012 (Norma Técnica Ecuatoriana INEN 2640:2012).

Los resultados obtenidos al final del ensayo de compostaje aerobio mostraron que el HDPE con aditivo $\mathrm{A} 1 \mathrm{al}$ canza el $75 \%$ de biodegradabilidad, tomando como referencia la celulosa, esto se debe a que el aditivo empleado se activa totalmente en condiciones de compostaje aerobio, debido a que es considerado un aditivo biodegradable; pero a los 60 días de ensayo, el aditivo A2 se activa, esto se puede observar en la Figura 4, donde la cantidad de $\mathrm{CO}_{2}$ acumulado sobrepasa al material con aditivo A3, este comportamiento se debe a la exposición a la humedad y temperatura, que activa el aditivo oxobiodegradable (Chiellini \& Solaro, 2003).

La celulosa alcanzó el $91 \%$ de biodegradabilidad respecto a la cantidad de $\mathrm{CO}_{2}$ que puede generar, lo cual se debe a que se trata de un material de origen vegetal y fácilmente bioasimilable en comparación al HDPE, tam- bién fue posible apreciar que las condiciones de compostaje suministradas fueron las indicadas, ya que el control positivo obtuvo el mayor valor de biodegradabilidad (San Andrés, 2010; Chiellini \& Solaro, 2003).

Los resultados alcanzados en los suelos utilizados para el compostaje, al finalizar el ensayo, revelaron un incremento en el contenido de cenizas, esto se debe al proceso de mineralización del suelo, y a que los materiales estudiados aportan con una cantidad de material inorgánico (Román et.al., 2013).

El contenido de materia orgánica y carbono orgánico muestran una disminución frente a los resultados iniciales, debido a que el carbono presente en el medio fue transformado en $\mathrm{CO}_{2}$, lo cual también explica la reducción de la relación $\mathrm{C} / \mathrm{N}$, que confirmó el proceso de descomposición.

Fue posible evidenciar que el $\mathrm{pH}$ del suelo aumentó luego del proceso de compostaje, lo que indica que no hubo formación significativa de ácidos grasos volátiles, lo que puede invalidar el ensayo por dar falsos positivos (Eldor, 2007). 


\section{CONCLUSIONES}

El HDPE A1 se consideró como un plástico compostable, de acuerdo con la norma NTE INEN 2643:2012, donde se toma como referencia a la celulosa como $100 \%$ de biodegradabilidad. Los porcentajes de biodegradabilidad obtenidos de los materiales de HDPE con aditivos A1, A2, A3 y celulosa por pérdida de masa y cantidad de $\mathrm{CO}_{2}$ que pueden generar teóricamente, confirman los resultados de generación de $\mathrm{CO}_{2}$ en 90 días.
Al finalizar el estudio se puede concluir que el uso de aditivos biodegradables se ha convertido en una posible alternativa para la protección del ambiente, pero es conveniente profundizar en el comportamiento microbiológico, para asegurar la descomposición de plásticos aditivados, así también en su posible fitotoxicidad. 


\section{LISTA DE REFERENCIAS}

Ammala, A. (2011). An overview of degradable and biodegradable polyolefins, Progress in polymer science, 36(8), 1015-1049.

APHA. (2005), AWWA and WEF Standard Methods for the Examination of Water \& Wastewater, 21 ed., American Public Health Association, Washington, D.C. Alcalinity Method 2320 B.

ASTM Standard D6954. (2004), Standard Guide for Exposing and Testing Plastics that Degrade in the Environment by a Combination of Oxidation and Biodegradation. West Conshohocken: ASTM International.

Carreira, D. (2011). Cuantificación de la Materia Orgánica del suelo. Método de WALKLEY \& BLACK, Jornadas de actualización: Gestión de la calidad en los laboratorios de análisis de suelos agropecuario. Argentina: SAMLA- PROINS, RILSAV.

Capuz, S. y Gómez, T. (2002). Ecodiseño $:$ Ingeniería del ciclo de vida para el desarrollo de productos sostenibles $\square$. Valencia: Universidad Politécnica de Valencia.

Chiellini, E. y Solaro, R. (2003), Biodegradable Polymers and Plastics. Pisa: University Pisa.

Ecuador al día. (2014). Ecuador produjo más de 1.400 millones de botellas plásticas en 2013. Recuperado de http://www.ecuadoraldia.com.ec/blog/2014/05/17/ecuadorprodujo-mas-de-1-400-millones-de-botellas-plasticas-en-2013

Eldor, P. (2007). Soil Microbiology, Ecology and Bochemistry. (3. ed.). Reino Unido: Elsevier Inc.

El Telégrafo. (2015). La industria plástica produce al menos \$ 418 millones al año. Recuperado de http://www.eltelegrafo.com.ec/noticias/economia/8/la-industria-plastica-produce-al-menos-418-millones-al-ano

FAO. (2003), On-farm Composting Methods. Rome: FAO. 
Kalil, P. (2007). Seguimiento del proceso de humificación en compost inoculado. Proyecto de grado Microbiología Industrial, Pontificia Universidad Javeriana, Bogotá D.C.

Ministerio de Agricultura, Ganadería, Acuacultura y Pesca. (2012). Provincia de Pichincha Mapa de Suelos - Taxonomía, Coordinación General del Sistema de Información Nacional - CGSIN. Recuperado de http://geoportal.agricultira.gob.ec/

Norma Técnica Ecuatoriana INEN 2640:2012. (2012) Método de Ensayo para Determinar la Biodegradabilidad Aeróbica de Materiales Plásticos Bajo Condiciones Controladas de Compostaje.

Román, P., Martínez, M. y Pantoja, A. (2013). Manual de compostaje del agricultor, Experiencias en América Latina. Santiago de Chile: Centro de Información de Recursos Naturales (CIREN).

San Andrés, M. (2010). Factores responsables de la degradación química de los polímeros. Efectos provocados por la radiación lumínica sobre algunos materiales utilizados en conservación: primeros resultados. Recuperado de http://ccfib.mcu. es/patrimonio/docs/MC/POLYEVART/FactrespXIReinaSof.pdf 